\section{Abdominal wall repair with human acellular dermal autograft}

\author{
Roel E. Genders, ${ }^{1}$ \\ Paul P.G.M. Kouwenberg, 2 \\ Rob P. Bleichrodt ${ }^{3}$
}

'Department of Dermatology, Leiden

University Medical Center, Netherlands;

2Department of Plastic Surgery,

Slingeland Hospital, Doetinchem,

Netherlands; ${ }^{3}$ Department of Surgery,

UMC St Radboud, Nijmegen, Netherlands

\section{Abstract}

Repair of abdominal wall defects in the presence of contamination or infection is a significant problem. The loss of tissue warrants enforcement of the abdominal wall, preferably by autologous material. However, autologous repair often requires extensive surgery. This paper presents a review of available literature of placement of an acellular human dermis to repair an abdominal fascia defect, in contaminated as well as in non-contaminated surgical fields. It is illustrated with a case report that describes the successful reconstruction of an infected abdominal wall defect with a human acellular dermis allograft. A systematic literature review was undertaken with searches performed in the Pubmed and Cochrane databases for the period up till March 2009, using the search terms Alloderm [Substance Name], Hernia [Mesh] and the key words acellular dermis, acellular dermal matrix, human acellular dermal allograft and abdominal wall defect. To assess methodological quality, each article was subjected to a modification of the methodological index for non-randomized studies (MINORS) according to Slim et al. Two items from the original index were not included because none of the studies selected had an unbiased assessment of the study end points and in none of the studies was a prospective calculation of the study size performed. Seventeen studies were included in the review. Data were extracted regarding study design, number of patients, surgical technique, followup period, contaminated or non-contaminated area of the fascia defect, mortality and morbidity (hemorrhage, seroma, wound dehiscence, infection) of the operative procedure, the longterm results (removal of the graft, reherniation and bulging) and level of evidencey. A total of 169 short-term complications and 151 longterm complications occurred after 643 surgical procedures reconstructing both contaminated and clean abdominal wall defects by implantation of an HADA. Human acellular dermal allo- graft seems to be a good alternative for autologous repair of contaminated or infected abdominal wall defects.

\section{Introduction}

Repair of abdominal wall defects in the presence of contamination or infection is a significant problem. The loss of tissue warrants enforcement of the abdominal wall preferably by autologous material. However, autologous repair often requires extensive operations that carry a high morbidity. ${ }^{1}$ Absorbable meshes are most often used under these circumstances. A major disadvantage, however, is the need for secondary reconstruction after the mesh has been absorbed. Human acellular dermal allograft (HADA) has been used for abdominal wall reconstruction under clean and contaminated conditions. The prosthesis is made out of human dermis that is processed to an acellular dermal graft without damage to the extra-cellular matrix. Alloderm ${ }^{\circledR}$ grafts are processed from human allograft skin and freeze-died during the process, resulting in an acellular dermal graft with an intact extra-cellular matrix. ${ }^{2}$ Alloderm ${ }^{\circledR}$ was first used in the treatment of full-thickness burn to prevent wound contraction and improve cosmesis. ${ }^{3}$ After implantation cellular infiltration, remodeling with autologous tissue and vascularisation occurs, which lessens the risk of infection. ${ }^{2,4-7}$

The use of HADA in a complicated case of abdominal wall infection with a successful post-operative course, led us to review the available literature about the repair of abdominal wall defects with human acellular dermis both under clean and contaminated conditions with respect to early (hemorrhage, seroma, wound dehiscence, infection) and late complications (bulging, herniation, re-operation).

\section{Case Report}

A 40-year-old woman underwent abdominal wall reconstruction with a polypropylene mesh after harvesting of an ipsilateral TRAM flap for breast reconstruction. The postoperative course was complicated by a wound infection that resulted in a chronic fistula and recurrent infections. Wound culture showed a hemolytic streptococcus group G. After one year the mesh was removed. The fascial defect was repaired with an inlay human acellular dermis (Alloderm $^{\circledR}$; LifeCell, Corp., Branchburg, NJ, USA) (Figure 1). The patient started a course of penicillin just before surgery and continued this for one week. The postoperative course was uneventful. At 18-month follow up there
Correspondence: Paul P.G.M. Kouwenberg, Department of Plastic Surgery, Slingeland Hospital, Kruisbergseweg 25, 7009 BL, Doetinchem, The Netherlands.

Tel. +31.314.329.944 - Fax: +31.314.329.946.

E-mail: p.kouwenberg@slingeland.nl

Key words: abdominal wall defect, infection, hernia, prosthesis related, AlloDerm, acellular dermis.

Acknowledgements: no funding, support or commercial interest interference

Received for publication: 19 July 2012.

Revision received: 9 November 2011.

Accepted for publication: 19 December 2011.

This work is licensed under a Creative Commons Attribution NonCommercial 3.0 License (CC BYNC 3.0).

(C) Copyright R.E. Genders et al., 2012

Licensee PAGEPress srl, Italy

Surgical Techniques Development 2012; 2:e6

doi:10.4081/std.2012.e6

were no signs of infection, hernia or bulging of the abdominal wall (Figure 2).

Histology of the removed prosthesis revealed polypropylene mesh that was fully incorporated in fibrocollagenous tissue. The tissue showed micro abscesses and was invaded by numerous polymorphonuclear granulocytes, macrophages and foreign body giant cells.

\section{Materials and Methods}

\section{Identification of studies}

A systematic review was undertaken with searches performed in the Pubmed and Cochrane databases for the period up till March 2009, using the search terms Alloderm [Substance Name], Hernia [Mesh] an the key words acellular dermis, acellular dermal matrix, human acellular dermal allograft and abdominal wall defect. Only English Language papers were considered. Experimental animal studies and case reports were excluded. Relevant publications about placing acellular dermis in an abdominal wall or fascia defect were acquired by assessing the titles and abstracts.

The manuscript was read and included in the study when appropriate. The references from these articles were tracked and screened first by reading the abstract and subsequently by reading the complete manuscript if necessary, and included in our study when appropriate. 


\section{Eligibility of studies}

To assess methodological quality, each article was subjected to a modification of the methodological index for non-randomized studies (MINORS) according to Slim et al. ${ }^{8}$ (Table 1). The MINORS index has been shown to be a valid instrument for assessing the methodological quality of non-randomized, comparative and non-comparative trials in surgery, developed for use by readers, manuscript reviewers or journal editors. Two items from the original index were not included because none of the studies selected had an unbiased assessment of the study end points and in none of the studies was a prospective calculation of the study size performed.

Each possible eligible article was scored independently by two of the authors (REG and PPGMK) as outlined in Table 1. The maximum score is 13 . To be selected, publications had to score at least 4 points on the items 1-6, reflect- ing an overall fair methodological quality and homogeneity. To determine reherniation rate, only studies that had a follow up of at least 12 months were included. Definite scores were established when consensus was reached between all authors.

\section{Data extraction}

After the initial assessment of the studies for eligibility the following data were extracted: study design, number of patients, surgical technique, follow up period, contaminated or non-contaminated area of the fascia defect, mortality and morbidity of the operative procedure, the long-term results (removal of the graft, reherniation and bulging) and level of evidence.

The desired homogeneity in representation of the adverse events was obtained by grouping complications in general categories as specified in the tables.

\section{Analysis}

The incidence of each item scored was calculated from the pooled patient data. For each specified item only those articles were used that present accurately described details on the specific item. For each item the total number of patients described in these articles is reported.

Statistical assessment was performed by using a $95 \%$ confidence interval.

\section{Results}

The literature search resulted in a total of 279 articles from PubMed and 78 from the Cochrane database. From the 357 abstracts studied, 15 met the inclusion criteria for this review. The related article search added 8 studies to be evaluated, leading to the inclusion of

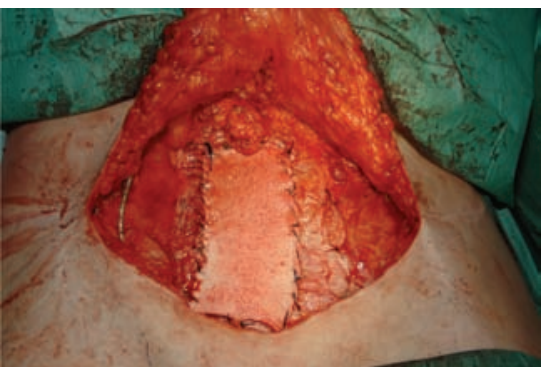

Figure 1. Operative field after implantation of the Alloderm ${ }^{\circledast}$ prosthesis. The prosthesis is sutured to the adjacent fascia with a running polydioxane suture 2.0 .

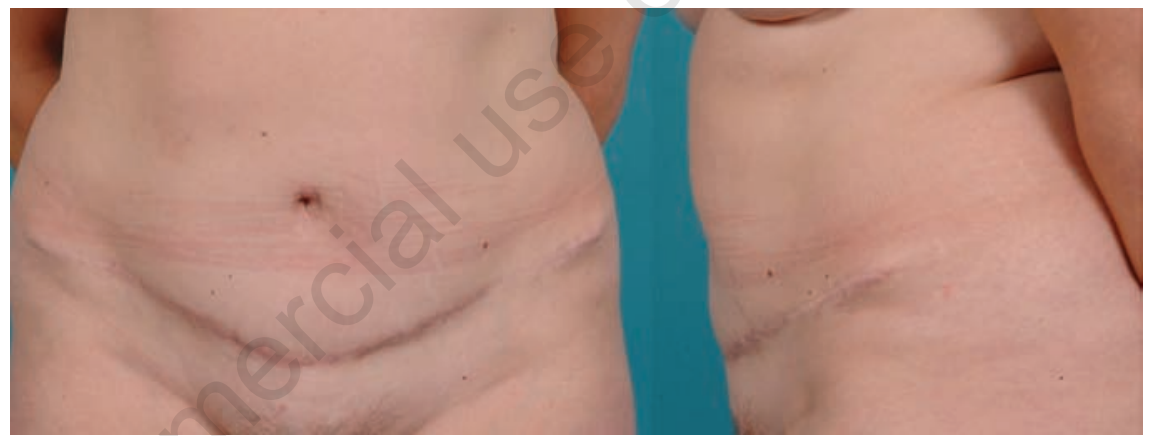

Figure 2. Result 18 months after implantation of an Alloderm ${ }^{\circledast}$ prosthesis. Frontal view (left). Lateral view (right).

Table 1. Modified methodological index of non-randomized trials according to the methodology of Slim $e t$ al. ${ }^{1}$ Since none of the publications reported unbiased assessment of the study end point, and none of the studies were prospective, these items that are originally part of MINORS were not included in the assessment of articles for inclusion.

$1 \quad$ A clearly stated aim: the question addressed should be precise and relevant in the light of available literature

2 Inclusion of consecutive patients: all patients potentially fit for inclusion (satisfying the criteria for inclusion) have been included in the study during the study period (no exclusion or details about the reasons for exclusion)

3 Endpoints appropriate to the aim of the study: unambiguous explanation of the criteria used to evaluate the main outcome which should be in accordance with the question addressed by the study. Also, the endpoints should be assessed on an intention-to-treat basis.

$4 \quad$ Unbiased assessment of the study endpoint: blind evaluation of objective endpoints and double-blind evaluation of subjective endpoints. Otherwise the reasons for not blinding should be stated

$5 \quad$ Follow up period appropriate to the aim of the study: the follow up should be sufficiently long to allow the assessment of the main endpoint and possible adverse events.

$6 \quad$ Loss to follow up less than 5\%: all patients should be included in the follow up. Otherwise, the proportion lost to follow up should not exceed the proportion experiencing the major endpoint

7 A follow up of at least 1-year to determine reherniation rate. This item is only used to select publications for determining reherniation rate.

\begin{tabular}{ll} 
Not reported & 0 \\
Partially reported & 1 \\
Clear aim & 2 \\
Not reported & 0 \\
Patients in certain time period & 1 \\
Consecutive patients + characteristics & 2 \\
\hline Not reported & 0 \\
Reported, but inadequately & 1 \\
Reported adequately & 2 \\
Item not reported & 0 \\
Reported, but inadequately & 1 \\
Reported adequately & 2 \\
Item not reported & 0 \\
Reported, but inadequately & 1 \\
Reported adequately & 2 \\
Item not reported & 0 \\
Reported, but inadequately & 1 \\
Reported adequately & 2 \\
No & 0 \\
Yes & 1 \\
\hline
\end{tabular}


another 2. Exclusion of articles having a MINORS score less than 1 point on one of the items scored, left 17 studies to be included in this systemic review: one retrospective casecontrol study and 16 case series (Figure 3 ).

The MINORS score of the case-control study was 10 . The median MINORS score of the 16 retrospective case series was 6 (range 4-10). Only 2 studies mentioned a protocolled prospective study design., ${ }^{9,10}$ The total number of patients treated with acellular human dermis (AHD) amounted to 643.

Five published studies were about the repair of abdominal wall defects under clean conditions, and 4 under contaminated conditions. Eight studies included both clean and contaminated cases. The surgical technique of AlloDerm placement included the onlay-, interposition-, underlay-placement of the graft and open wound treatment in assistance with vacuum assisted therapy (Table 2).

\section{Postoperative mortality and morbidity}

Mortality was reported in 6 studies and included 10 patients. (11-16) In 3 cases it was explicitly reported as postoperative mortality (mortality within thirty days of the in-hospital mortality) due to complicated co-morbidity.

The other 7 patients were mentioned as patients who died during the follow up 3-10 months after surgery, and their death was not related to the placement of AlloDerm but due to co-morbidity. In the remaining studies, no patients died after surgery or during follow up. The overall postoperative mortality was $0.47 \%$ (95\% CI 0-0.99\%).
Overall postoperative morbidity was $27.9 \%$ (95\% CI 31-24\%, range 0-54\%) in 606 patients because one study only discussed herniation and bulging rates but no postoperative morbidity rates (Tables 2 and 3). Wound infections were found in 96 of 606 patients $15.8 \%$ (95\%
CI 12.9-18.7\%, range 0-41\%). Hemorrhage was observed in $1.3 \%$ (95\% CI $0.2-2.4 \%$, range 2 $14 \%)$. The forming of seroma was noted in $10.5 \%$ (95\% CI 7.7-13.4\%; range 0-27\%). Wound dehiscence occurred in 20 of 405 patients (4.9\%; 95\% CI 2.8-7.0\%; range 0-15\%).

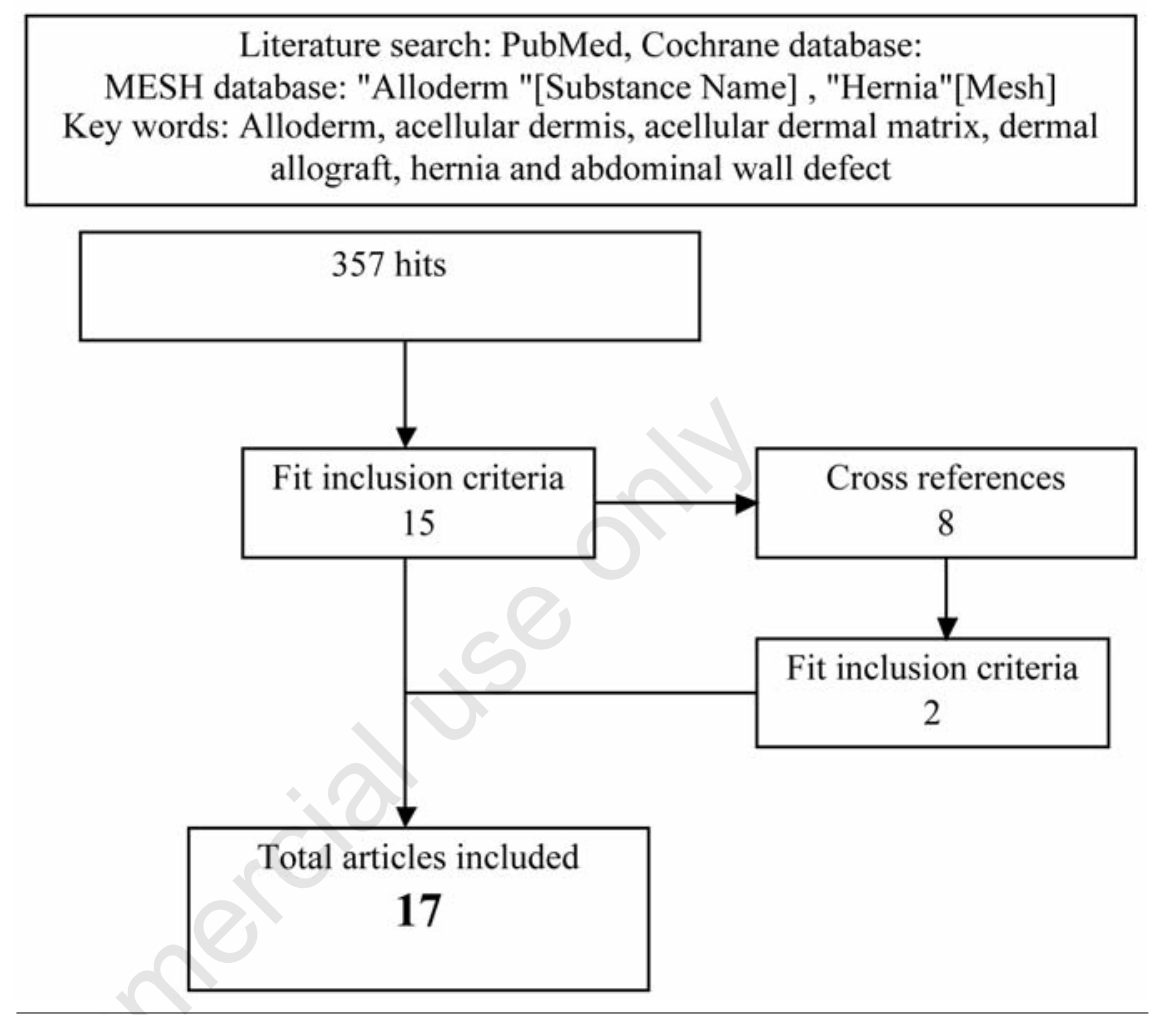

Figure 3. Research inclusion criteria.

Table 2. The surgical technique of AlloDerm placement described in the literature.

\begin{tabular}{|c|c|c|c|c|c|c|}
\hline Study & Minors & $\begin{array}{l}\text { Level of } \\
\text { evidence }\end{array}$ & $\begin{array}{l}\text { Follow up } \\
\text { (months) }\end{array}$ & Operation technique & $\begin{array}{l}\text { Clean/contaminated } \\
\text { surgery field }\end{array}$ & n \\
\hline Buinewicz $\mathrm{B}^{4}$ & 6 & 4 & 20 & Interposition, onlay & clean & 44 \\
\hline Kolker AR ${ }^{17}$ & 6 & 4 & 16 & In combination with $\mathrm{CST}^{*}$ & both & 16 \\
\hline Glasberg SB ${ }^{9}$ & 8 & 4 & 19 & Inlay & clean & 54 \\
\hline Diaz JJ'14 & 6 & 4 & 9 & Inlay/interposition, onlay & both & 75 \\
\hline Butler CE ${ }^{12}$ & 5 & 4 & 6 & Interposition, onlay & both & 13 \\
\hline Albo D ${ }^{11}$ & 4 & 4 & 9 & Inlay & contaminated & 12 \\
\hline Schuster $\mathrm{R}^{16}$ & 5 & 4 & 9 & Inlay & contaminated & 18 \\
\hline Kim $\mathrm{H}^{15}$ & 4 & 4 & 6 & Inlay & both & 29 \\
\hline Patton JH Jr ${ }^{18}$ & 4 & 4 & 11 & Inlay/interposition, onlay & contaminated & 67 \\
\hline Gupta $\mathrm{A}^{10}$ & 10 & 4 & 18 & Interposition & clean & 33 \\
\hline Espinosa-de-los-Monteros A ${ }^{19}$ & 10 & 3 & 13 & Onlay & clean & 39 \\
\hline Bellows $\mathrm{CF}^{20}$ & 7 & 4 & 9 & Inlay & both & 20 \\
\hline Jin $J^{21}$ & 8 & 4 & 21 & Inlay & both & 37 \\
\hline Lipman J22 & 5 & 4 & 10 & Underlay, onlay i.c.w. CST & both & 7 \\
\hline Misra $\mathrm{S}^{23}$ & 8 & 4 & 12 & Underlay, inlay, onlay & clean & 70 \\
\hline Candage $\mathrm{R}^{13}$ & 9 & 4 & 12 & Underlay, inlay, onlay & both & 26 \\
\hline Maurice $\mathrm{SM}^{24}$ & 7 & 4 & 7 & Underlay, inlay, onlay & both & 63 \\
\hline
\end{tabular}

"CTS, components separation technique. 


\section{Long-term follow up}

A mean follow up of at least one year was reported in 8 studies for a total of 339 patients (Table 4). Reherniation was found in $13.0 \%$ (95\% CI 9.4-16.6\%; range 0-32\%) and bulging in $12.2 \%$ ( $95 \%$ CI $8.5-15.9 \%$; range $0-45 \%$ ) of the patients. Removal of the HADA due to rejection was reported in one patient.

In the nine studies with a mean follow up of less than one year, reoperation was mentioned in 17 cases, due to irreversible damage to the allograft. In these studies, 69 reherniations (22.7\%; 95\% CI 18.0-27.4\%; range 0-50\%) and 2 bulgings $(0.7 \%$; range $0-10 \%)$ were observed among 304 patients.

There were only two prospective studies in which all data were collected prospectively from consecutive patients with ventral hernia repair performing HADA implantation by the interposition technique in a clean operation field.9,10 There were no patients lost to follow up; the mean follow up period was 18 months in both studies. There were 54 complications reported in 87 operated patients. Sixteen patients developed a seroma, which was treated conservatively, 3 patients developed a wound dehiscence, and no infections were seen. Twenty-seven patients developed problems of diastase and abdominal bulging, with operative correction in 8 patients. Eight reherniations with consecutive reoperations were observed.

\section{Discussion}

A growing number of patients surviving intra-abdominal sepsis have a combination of an abdominal wall defect with chronic infection or fistulas. Bridging the fascial gap with prosthetic material is still the most frequently applied method of reconstruction, but implantation of biomaterials is accompanied by an increased risk of visceral damage and infection, particularly when they are used for reconstruction in a contaminated environment. ${ }^{29-31}$

Suture repair can only be used in hernias that can be closed primarily. However, suture repair either performed under clean or contaminated conditions is accompanied by an unacceptable recurrence rate of up to $45 \% .{ }^{32-34,36} \mathrm{To}$ prevent reherniation, HADA is used to strengthen the reconstruction, either by onlay or sublay implantation. Espinosa-de-losMonteros et al. retrospectively evaluated the outcome of patients treated with human cadaveric acellular dermis as an adjunct to abdominal wall reconstruction and compared the results with historical controls. HADA significantly decreased recurrence rates in patients with medium-sized hernias. No differences were observed when adding human cadaveric acellular dermis as an overlay to patients with

Table 3. Overall postoperative morbidity.

\begin{tabular}{lcc} 
Short term complications & & n \\
Infection ref(4;9-20;25-27) & $96 / 606$ & $0.16+/-0.029$ \\
Dehiscence ref(4;9;10;12-14;17;19;20;25-27) & $20 / 405$ & $0.049+/-0.021$ \\
\hline Seroma ref(4;9-13;15;17;19;20;25-27) & $47 / 446$ & $0.11+/-0.028$ \\
Haemorrhage ref(12;13;15;19;25;26) & $6 / 446$ & $0.013+/-0.011$ \\
\hline TOTAL & $169 / 606$ & $0.31+/-0.24$ \\
\hline
\end{tabular}

Table 4. Long-term follow up.

\begin{tabular}{lcc}
\hline Long-term complications & Follow up $>1$ year & \\
& n & $\%(95 \% \mathrm{Cl})$ \\
\hline Bulging $(9 ; 10 ; 13 ; 17 ; 19 ; 27 ; 28)$ & $36 / 295$ & $0.12+/-0.037$ \\
Hernia $(4 ; ; ; 10 ; 13 ; 17 ; 19 ; 27 ; 28)$ & $44 / 339$ & $0.13+/-0.036$ \\
\hline
\end{tabular}

large sized hernias treated with underlay mesh. The use of human cadaveric acellular dermis did not increase postoperative morbidity rates.

If primary closure is impossible, the fascial gap must be bridged by bioabsorbable meshes, autologous material, or collagen based prosthetic materials. Application of bioabsorbable meshes such as Polyglactin mesh inevitably results in herniation within 3-6 months after implantation. Recently, autologous repair techniques have been reviewed by de Vries Reilingh et al. ${ }^{1}$ The Components Separation Technique is the best documented procedure. The operation requires extensive dissection and is associated with a morbidity of $24.0 \%$ and a recurrence rate of $18.2 \%$. Although the results of the da Silva technique using both the anterior and posterior rectus sheaths to bridge the fascial gap are good (morbidity $5-20 \%$ and reherniation 0-3\%), the poor methodological quality of the studies precludes firm conclusions. Repair with free fascia lata or dermal grafts is an alternative if the above techniques cannot be used. However, wound complications are frequent (42\%) and recurrent hernias are reported in up to $29 \%$. Pedicled or free vascularized flaps are reserved for complex situations only.

Biological materials theoretically may provide a good solution for hernia repair in a contaminated field. The most commonly used biological materials are HADA, porcine dermal collagen (PDC), small intestinal submucosa (SIS), bovine pericardium and human dura mater. Reabsorption of the collagen matrix, resulting in bulging and reherniation are the major problems of these prosthesis.

HADA has been used extensively for hernia repair. The results of treatment of more than 500 patients have been published in literature. It is thought that the prosthesis is a scaffold for the in growth of fibrocollagenous tissue, and that most of the biomaterial stays in place.
Breakdown and resorption is prevented by cross linking of the collagen fibres which makes them resistant to proteolytic enzymes. The biomaterial is acellular and does not provoke an immunogenic reaction. No adverse responses have been reported in the 17 publications that were reviewed by us, including 643 patients. The methodological quality of the publications was however poor and precludes firm conclusions and makes comparison with other techniques hazardous.

HADA is used to strengthen primary fascial closure as a sublay or onlay, or to bridge a fascial gap. It is used under clean and contaminated conditions and even in the presence of overt infection. The short-term results with HADA to bridge fascial defects are similar to those found in autologous repair. Wound infections are reported in $13 \%$ of patients and $6.4 \%$ fascial dehiscences. The long-term results are similar when only studies with a follow up of at least one year are taken into account. Reherniation rates after a follow up of at least one year are $13 \%$ after excluding patients with bulging (11\%) and fascial dehiscence (6.4\%). Studies with a shorter follow up reported a recurrence rate of $22.7 \%$ of patients. In these studies with shorter follow up, more surgical interventions were performed in a contaminated operative field (Table 2). But because also different surgical techniques were used, a hard conclusion could not be drawn. The same problem was encountered with the differences in onlay, inlay or sublay placing of the HADA. Therefore, HADA repair cannot be advocated as an alternative for CST and the da Silva technique. However, these techniques can only be used to repair midline hernias.

Repair of other hernias can be performed with HADA, free fascia lata grafts, or pedicled or free vascularized flaps. Sufficient data are not available to compare the results of HADA and pedicled flaps. However, donor site compli- 
cations are a potential disadvantage of autologous repair. Free fascia lata grafts are the most often used free fascial transplants. These grafts are incorporated into the adjacent study and remain viable as a result of early revascularization. ${ }^{1}$ Donor site complications occur in $13-17 \%$ and recurrent hernias in $9-25 \%$ after a follow up of at least one year. ${ }^{37-39}$ As in free fascial grafts, acellular dermal grafts show rapid vascularization and in growth of fibrocollagenous tissue. The prevention of donor site complications is a potential advantage of HADA over free fascia lata grafts., 2,-7,40,41

The literature studied does not justify the routine use of HADA to repair abdominal wall hernias and defects. However, the quality of the available literature is poor due to lack of study-protocols, inadequate follow up and adequate definitions of the end points. The diversity of used techniques to implant HADA, its use under clean or contaminated conditions, and the great variety of hernias and defects (all important factors that influence outcome) further compromise the interpretation of the results. The indications for HADA are, therefore, limited to repairs for which no standard technique using autologous tissue is available, or in cases in which the disadvantages of repair outweigh those of HADA repair, including the prevention of donor-site complications.

When HADA is used, the outcome seems to be affected by contamination of the operative area, although evidence is too poor to be statistically significant. Moreover, implantation by the inlay technique is associated with an increased recurrence rate. Jin et al. retrospectively studied patients with abdominal defects repaired with HADA and compared two techniques, fascial bridging versus fascial reinforcement repair, with regard to their longterm recurrence rates. ${ }^{28}$ It was concluded that HADA should be used only as reinforcement after primary fascial reapproximation because recurrence rate in patients in whom the prosthesis is used to bridge the fascial gap is very high. From our collected data, we were not able to stratify the outcome by the operation technique because of the lack of necessary data. One study reported no difference in outcome between onlay and inlay operation techniques. ${ }^{14}$ Another study made a division into two subgroups, with the second subgroup reporting more taut suturing of the HADA in the fascia defect, based on their former experiences. In the first subgroup there was little experience with HADA and it suturing did not adequately fit the defect, resulting in more seromas and bulging of the abdominal wall than the second subgroup. ${ }^{9}$

Repair of abdominal wall hernias or defects with human acellular dermis allograft (HADA) is a treatment modality for which ample evidence is present in literature. The results are similar to autologous repair techniques with respect to postoperative morbidity, but the long-tem results are worse (reherniation, graft removal and bulging). The level of evidence is, however, low, since this conclusion is mainly based on retrospective case series. Prospective studies are needed to determine the place of HADA in the repair of contaminated abdominal wall hernias.

\section{References}

1. de Vries Reilingh TS, Bodegom ME, van $\mathrm{GH}$, et al. Autologous tissue repair of large abdominal wall defects. Br J Surg 2007; 94:791-803.

2. Livesey SA, Herndon DN, Hollyoak MA, et al. Transplanted acellular allograft dermal matrix. Potential as a template for the reconstruction of viable dermis. Transplantation 1995;60:1-9.

3. Wainwright DJ. Use of an acellular allograft dermal matrix (AlloDerm) in the management of full-thickness burns. Burns 1995;21:243-8.

4. Buinewicz B, Rosen B. Acellular cadaveric dermis (AlloDerm): a new alternative for abdominal hernia repair. Ann Plast Surg 2004;52:188-94.

5. Butler CE, Prieto VG. Reduction of adhesions with composite AlloDerm/polypropylene mesh implants for abdominal wall reconstruction. Plast Reconstr Surg 2004;114:464-73.

6. Eppley BL. Revascularization of acellular human dermis (alloderm) in subcutaneous implantation. Aesthet Surg J 2000; 20:291-5.

7. Menon NG, Rodriguez ED, Byrnes CK, et al. Revascularization of human acellular dermis in full-thickness abdominal wall reconstruction in the rabbit model. Ann Plast Surg 2003;50:523-7.

8. Slim K, Nini E, Forestier D, et al. Methodological index for non-randomized studies (minors): development and validation of a new instrument. ANZ J Surg 2003;73:712-6.

9. Glasberg SB, D'Amico RA. Use of regenerative human acellular tissue (AlloDerm) to reconstruct the abdominal wall following pedicle TRAM flap breast reconstruction surgery. Plast Reconstr Surg 2006;118:815.

10. Gupta A, Zahriya K, Mullens PL, et al. Ventral herniorrhaphy: experience with two different biosynthetic mesh materials, Surgisis and Alloderm. Hernia 2006;10: 419-25.

11. Albo D, Awad SS, Berger DH, Bellows CF. Decellularized human cadaveric dermis provides a safe alternative for primary inguinal hernia repair in contaminated surgical fields. Am J Surg 2006;192:e12-7.

12. Butler CE, Langstein HN, Kronowitz SJ. Pelvic, abdominal, and chest wall reconstruction with AlloDerm in patients at increased risk for mesh-related complications. Plast Reconstr Surg 2005;116:126375.

13. Candage R, Jones $\mathrm{K}$, Luchette FA, et al. Use of human acellular dermal matrix for hernia repair: friend or foe? Surgery 2008;144:703-9.

14. Diaz JJ Jr, Guy J, Berkes MB, et al. Acellular dermal allograft for ventral hernia repair in the compromised surgical field. Am Surg 2006;72:1181-7.

15. Kim H, Bruen K, Vargo D. Acellular dermal matrix in the management of high-risk abdominal wall defects. Am J Surg 2006; 192:705-9.

16. Schuster R, Singh J, Safadi BY, Wren SM. The use of acellular dermal matrix for contaminated abdominal wall defects: wound status predicts success. Am J Surg 2006; 192:594-7.

17. Kolker AR, Brown DJ, Redstone JS, et al. Multilayer reconstruction of abdominal wall defects with acellular dermal allograft (AlloDerm) and component separation. Ann Plast Surg 2005;55:36-41.

18. Patton JH Jr, Berry S, Kralovich KA. Use of human acellular dermal matrix in complex and contaminated abdominal wall reconstructions. Am J Surg 200;193:360-3.

19. Espinosa-de-los-Monteros A, de la Torre JI, Marrero I, et al. Utilization of human cadaveric acellular dermis for abdominal hernia reconstruction. Ann Plast Surg 2007;58:264-7.

20. Bellows CF, Albo D, Berger DH, Awad SS. Abdominal wall repair using human acellular dermis. Am J Surg 2007;194:192-8.

21. Jin J, Rosen MJ, Blatnik J, et al. Use of acellular dermal matrix for complicated ventral hernia repair: does technique affect outcomes? J Am Coll Surg 2007; 205:654-60.

22. Lipman J, Medalie D, Rosen MJ. Staged repair of massive incisional hernias with loss of abdominal domain: a novel approach. Am J Surg 2008;195:84-8.

23. Misra S, Raj PK, Tarr SM, Treat RC. Results of AlloDerm use in abdominal hernia repair. Hernia 2008;12:247-50.

24. Maurice SM, Skeete DA. Use of human acellular dermal matrix for abdominal wall reconstructions. Am J Surg 2009;197:3542.

25. Lipman J, Medalie D, Rosen MJ. Staged repair of massive incisional hernias with loss of abdominal domain: a novel approach. Am J Surg 2008;195:84-8.

26. Maurice SM, Skeete DA. Use of human acellular dermal matrix for abdominal wall reconstructions. Am J Surg 2009;197:35- 
42.

27. Misra S, Raj PK, Tarr SM, Treat RC. Results of AlloDerm use in abdominal hernia repair. Hernia 2008;12:247-50.

28. Jin J, Rosen MJ, Blatnik J, et al. Use of acellular dermal matrix for complicated ventral hernia repair: does technique affect outcomes? J Am Coll Surg 2007; 205:654-60.

29. Dayton MT, Buchele BA, Shirazi SS, Hunt LB. Use of an absorbable mesh to repair contaminated abdominal-wall defects. Arch Surg 1986;121:954-60.

30. Gilbert AI, Felton LL. Infection in inguinal hernia repair considering biomaterials and antibiotics. Surg Gynecol Obstet 1993; 177:126-30.

31. Korenkov M, Sauerland S, Arndt M, et al. Randomized clinical trial of suture repair, polypropylene mesh or autodermal hernioplasty for incisional hernia. Br J Surg 2002;89:50-6.

32. Lallement M, Missana MC, Bourgeon Y,
Abbes M. Closure of the abdominal wall after removal of a mucocutaneous flap from the transverse rectus abdominis for breast reconstruction. Apropos of 48 cases. Review of the literature. Ann Chir Plast Esthet 1994;39:733-41.

33. Petit JY, Rietjens M, Garusi C, et al. Abdominal complications and sequelae after breast reconstruction with pedicled TRAM flap: is there still an indication for pedicled TRAM in the year 2003? Plast Reconstr Surg 2003;112:1063-5.

34. Goris JA. Ogilvie's method applied to infected wound disruption. Arch Surg 1980;115:1103-7.

35. Kendrick JH, Casali RE, Lang NP, Read RC. The complicated septic abdominal wound. Arch Surg 1982;117:464-8.

36. Stone HH, Fabian TC, Turkleson ML, Jurkiewicz MJ. Management of acute fullthickness losses of the abdominal wall. Ann Surg 1981;193:612-8.

37. Disa JJ, Goldberg NH, Carlton JM, et al.
Restoring abdominal wall integrity in contaminated tissue-deficient wounds using autologous fascia grafts. Plast Reconstr Surg 1998;101:979-86.

38. Sukkar SM, Dumanian GA, Szczerba SM, Tellez MG. Challenging abdominal wall defects. Am J Surg 2001;181:115-21.

39. Williams JK, Carlson GW, deChalain T, et al. Role of tensor fasciae latae in abdominal wall reconstruction. Plast Reconstr Surg 1998;101:713-8.

40. Lattari V, Jones LM, Varcelotti JR, et al. The use of a permanent dermal allograft in full-thickness burns of the hand and foot: a report of three cases. J Burn Care Rehabil 1997;18:147-55.

41. Reagan BJ, Madden MR, Huo J, et al. Analysis of cellular and decellular allogeneic dermal grafts for the treatment of full-thickness wounds in a porcine model. J Trauma 1997;43:458-66. 